\title{
Notificações de surtos de doenças veiculadas por alimentos no Estado de São Paulo, no período de 2011 a 2018
}

\author{
Notifications of outbreaks of foodborne diseases in the State of São Paulo, from 2011 to \\ 2018
}

\section{Ludimila dos Santos Antônio', Bruna Maria Salotti de Souza², Luis Antônio Mathias ${ }^{3}$}

Resumo: Consideradas amplo problema de Saúde Pública, as Doenças Veiculadas por Alimentos - DVA são causadas pela ingestão de alimento ou água contaminados. Um surto de DVA é caracterizado pela presença da doença em duas ou mais pessoas. No Brasil, o Estado de São Paulo possui o maior número de surtos de DVA notificados. O presente trabalho teve como objetivo, realizar o levantamento das notificações de surtos de DVA no Estado de São Paulo no período de 2011 a 2018. Nos oito anos analisados foram confirmados 1.112 surtos de DVA, um total de 74.268 consumidores foram expostos ao risco, 33.377 consumidores desenvolveram doença e 11 óbitos foram registrados. A maioria dos surtos confirmados ocorreram na região metropolitana de São Paulo - SP. Os locais de maior ocorrência de surtos de DVA foram creches e escolas, residências e estabelecimentos comercializadores de alimentos. Os alimentos mais envolvidos nos surtos foram massas, água e produtos cárneos, porém a transmissão direta pessoa a pessoa foi responsável pela maioria dos casos. Os agentes etiológicos mais envolvidos nos surtos foram Norovírus e Rotavírus, considerando número de casos e surtos, e Salmonella spp., Escherichia coli e Shigella spp., considerando número de óbitos. Informações importantes estão incompletas na base de dados, o que prejudica o conhecimento sobre evolução dos surtos de DVA no Estado de São Paulo.

Palavras chave: contaminação; Escherichia coli, Norovírus; Salmonella spp. e Shigella spp..

Abstract: Considered a broad public health problem, Foodborne Diseases - FVB are caused by eating contaminated food or water. An outbreak of FVB is characterized by the presence of the disease in two or more people. In Brazil, the State of São Paulo has the highest number of reported FVB outbreaks. This study aimed to survey the reports of FVB outbreaks in the State of São Paulo in the period from 2011 to 2018. In the eight years analyzed, 1,112 FVB outbreaks, a total of 74,268 consumers were exposed to the risk, 33,377 consumers developed the disease and 11 deaths were recorded. Most of the confirmed outbreaks occurred in the metropolitan region of São Paulo - SP. The places with the highest occurrence of FVB outbreaks were day care centers and schools, homes and food merchants. The foods most involved in the outbreaks were pasta, water and meat products, but direct person-to-person transmission was responsible for most cases. The etiological agents most involved in the outbreaks were Norovirus and Rotavirus, considering the number of cases and

\section{http://dx.doi.org/}

Autor para correspondência. E-mail: ludy-40@live.com

Recebido em 15.02.2021. Aceito em 30.06.2021

${ }^{1}$ Residente no Programa de Residência em Área Profissional da Saúde - Medicina Veterinária e Saúde - UNESP - Universidade Estadual Paulista, Jaboticabal, SP, Brasil - ludy-40@live.com

${ }^{2}$ Doutora, UFMG - Universidade Federal de Minas Gerais, Departamento de Tecnologia e Inspeção de Produtos de Origem Animal, Belo Horizonte, MG, Brasil - brunasouza@ vetufmg.edu.br

${ }^{3}$ Doutor, UNESP - Universidade Estadual Paulista, Jaboticabal, SP, Brasil - la.mathias@ unesp.br 
outbreaks, and Salmonella spp., Escherichia coli and Shigella spp., considering the number of deaths. Important information is incomplete in the database, which hinders the knowledge about the evolution of FVB outbreaks in the State of São Paulo.

Keywords: contamination; Escherichia coli, Norovírus; Salmonella spp. e Shigella spp..

\section{Introdução}

O conceito de saúde única compreende a união indissociável das saúdes humana, animal e ambiental. O combate às Doenças Veiculadas por Alimentos (DVA) tem papel importante neste conceito, uma vez que, por meio da segurança de alimentos, se tornou parte de um dos três pilares essenciais à promoção da saúde única, juntamente com o combate à resistência aos antimicrobianos e o controle de zoonoses (Cirne \& Cabrera, 2020).

A Organização Mundial de Saúde (OMS) considera as DVA uma questão importante de saúde pública global. Segundo a referida organização, estima-se que cerca de 600 milhões de pessoas adoeçam após a ingestão de alimentos contaminados e 420 mil óbitos sejam registrados anualmente em todo o mundo. De acordo com a Organização PanAmericana da Saúde, a ocorrência de DVA, além de sobrecarregar os sistemas de saúde, também impede o desenvolvimento socioeconômico, prejudicando as economias nacionais, o turismo e o comércio (WHO, 2015).

Devidamente considerado um Evento de Saúde Pública (ESP) que pode representar ameaça à saúde, um surto de DVA é caracterizado pela presença de doença similar em duas ou mais pessoas, após a ingestão de água ou alimento contaminado. A preocupação com tais doenças tem aumentado no Brasil e no mundo, fato que exige maior fiscalização das vigilâncias em saúde e das empresas que produzem e comercializam alimentos (AMARAL et al., 2019).

A ocorrência de DVA relaciona-se com diversos fatores, como: condições impróprias de saneamento e da qualidade da água para consumo humano; práticas inadequadas de higiene pessoal e consumo de alimentos contaminados. O contato do alimento com fezes durante sua produção, a falta de cuidados em seu armazenamento, a falta de higiene do manipulador e a utilização de água sem tratamento são exemplos de alguns dos potenciais que aumentam a probabilidade de contaminação do alimento (Brasil, 2019).

Sabe-se que a contaminação dos alimentos pode acontecer por meio de perigos químicos, físicos ou biológicos, e que a ingestão do alimento contaminado pode acarretar o desenvolvimento de doenças. Entre os exemplos de contaminantes de natureza química estão 
metais pesados, agrotóxicos, antibióticos e toxinas de animais ou plantas; os físicos podem ser pedaços de vidro ou fragmentos metálicos; e a contaminação de natureza biológica, considerada a mais importante para a saúde pública, é atribuída a bactérias, vírus, fungos e parasitas (SANTOS, 2019).

Dentre os mais de 250 tipos de DVA existentes em todo o mundo, a maioria delas são doenças causadas por bactérias e suas toxinas, vírus e outros parasitas. No Brasil, a maioria dos surtos de DVA são causados por bactérias, principalmente por Salmonella spp., Escherichia coli $e$ Staphylococcus spp.. No entanto, há também surtos de DVA causados por vírus (rotavírus e norovírus) e, em menor proporção, por substâncias químicas (Brasil, 2019).

Comumente, as causas de surtos de DVA são atribuídas à ingestão de alimentos sem alteração sensorial perceptível, que os caracterize como alimentos fonte de DVA. Alimentos com aparência ou odor alterados comumente são rejeitados pelos consumidores e, dessa forma, dificilmente causam surtos alimentares, o que dificulta a investigação dos alimentos envolvidos nesses eventos, uma vez que os consumidores afetados geralmente não são capazes de identificar sensorialmente os alimentos contaminados (MARINHO et al., 2015).
Apesar da incidência de DVA crescer todos os anos, a maioria dos casos não é notificada, isso porque geralmente os indivíduos afetados apresentam discretos quadros de diarreia e vômito, e por este motivo não procuram pelos serviços de saúde. Em alguns desses casos, o paciente também associa a ligeira indisposição com sintomas gripais, e não procura atendimento médico especializado. Tais fatores, associados à demora para o diagnóstico, a multiplicidade de agentes etiológicos e a falta de conhecimento sobre o alimento envolvido no surto, contribuem para a subnotificação dos casos (AMARAL et al., 2019).

Hoje reconhecida como um problema amplo de saúde pública, a segurança sanitária dos alimentos passou a representar um desafio para profissionais da área da saúde, no que diz respeito à definição de meios efetivos de proteção à saúde das populações, incluindo o fortalecimento dos sistemas das vigilâncias. No Brasil, a Vigilância Epidemiológica das DVA (VE-DVA) monitora os surtos de DVA e os casos das doenças relacionadas ao consumo de alimentos. De acordo com dados do Sistema de Informação de Agravos de Notificação (SINAN), são notificados em média, por ano, 700 surtos de DVA no país (Brasil, 2019). 
O desempenho do Sistema Nacional de Vigilância Epidemiológica das DVA nos diferentes estados do Brasil ainda é heterogêneo. Em decorrência da fragilidade dos programas de controle da qualidade de alimentos existentes ou da ausência de sistemas de vigilância sanitária, as informações existentes a respeito das DVA não representam a real magnitude do problema no país. Situação já enfrentada por muitos países, nos quais o número real de DVA teve uma frequência 300 a 350 vezes maior do que indicam os relatos oficiais (Melo et al., 2018). Neste sentido, o presente trabalho teve como objetivo realizar o levantamento das notificações de surtos de doenças veiculadas por alimentos no Estado de São Paulo, no período de 2011 a 2018, a fim de identificar as regiões do Estado e os locais com maior ocorrência de surtos de DVA; identificar os alimentos e os agentes etiológicos envolvidos nos surtos; e caracterizar os subgrupos populacionais de maior risco, de acordo com idade e sexo.

\section{Material e Métodos}

Realizou-se uma pesquisa documental por meio de um estudo retrospectivo descritivo. O levantamento dos dados epidemiológicos sobre a ocorrência de DVA no Estado de São
Paulo no período de 2011 a 2018 foi obtido a partir da base de dados do Centro de Vigilância Epidemiológica "Prof. Alexandre Vranjac", da Secretaria Estadual de Saúde do Estado de São Paulo (CVESP).

Foram avaliados os boletins dos municípios que compõem todos os 17 Departamentos Regionais de Saúde (DRS), da Secretaria Estadual de Saúde do Estado de São Paulo, Brasil, englobando os surtos alimentares registrados.

As DRS são divididas em: DRS I Grande São Paulo, DRS II - Araçatuba, DRS III - Araraquara, DRS IV - Baixada Santista, DRS V - Barretos, DRS VI Bauru, DRS VII - Campinas, DRS VIII Franca, DRS IX - Marília, DRS X Piracicaba, DRS XI - Presidente Prudente, DRS XII - Registro, DRS XIII - Ribeirão Preto, DRS XIV - São João da Boa Vista, DRS XV - São José do Rio Preto, DRS XVI - Sorocaba, DRS XVII - Taubaté.

Os dados referentes aos surtos de DVA notificados ao CVE-SP foram processados e condensados no programa Excel for Windows versão 16.0 $\left(\right.$ Microsoft $^{\circledR}$ ) e os resultados discriminados por data (ano), número de surtos, número de expostos (pessoas expostas ao alimento contaminado), número de casos (pessoas doentes), número de óbitos, local e 
município de ocorrência, agente etiológico, fonte de transmissão (alimentos envolvidos), idade e sexo dos consumidores afetados.

Os dados da população foram obtidos na base de dados do Instituto Brasileiro de Geografia e Estatística IBGE. Para cálculo das taxas de incidência ano a ano de casos de DVA no Estado de São Paulo, foram consideradas as variações anuais da população no período. Para cálculo das taxas de incidência por DRS e por faixas etárias, foram considerados os dados da população disponibilizados pelo último censo demográfico realizado em 2010.

Analisou-se a tendência de longo prazo da taxa incidência de casos anuais de DVA por meio de um modelo de regressão de Prais-Winsten (DOHOO et al., 2010).

A análise foi realizada utilizando o pacote "prais" do software R.

\section{Resultados e Discussão}

No período de 2011 a 2018 foram confirmados 1.112 surtos de DVA no Estado de São Paulo. Um total de 74.268 consumidores foram expostos ao risco, 33.377 consumidores desenvolveram doença e 11 óbitos foram registrados, gerando uma taxa de ataque da doença de $44,9 \%$ e uma taxa de letalidade de $0,03 \%$. Além dos surtos confirmados, no mesmo período foram notificadas 2.375 suspeitas de surtos de DVA, que podem ter afetado mais 40.807 consumidores.

Observa-se na Figura 1 que o número de suspeitas de surtos de DVA notificadas no Estado de São Paulo no período 2011 a 2018 corresponde a mais que o dobro dos surtos confirmados no Estado no mesmo período, o que gera dúvidas da verdadeira proporção da problemática e do real número de surtos de DVA ocorridos.

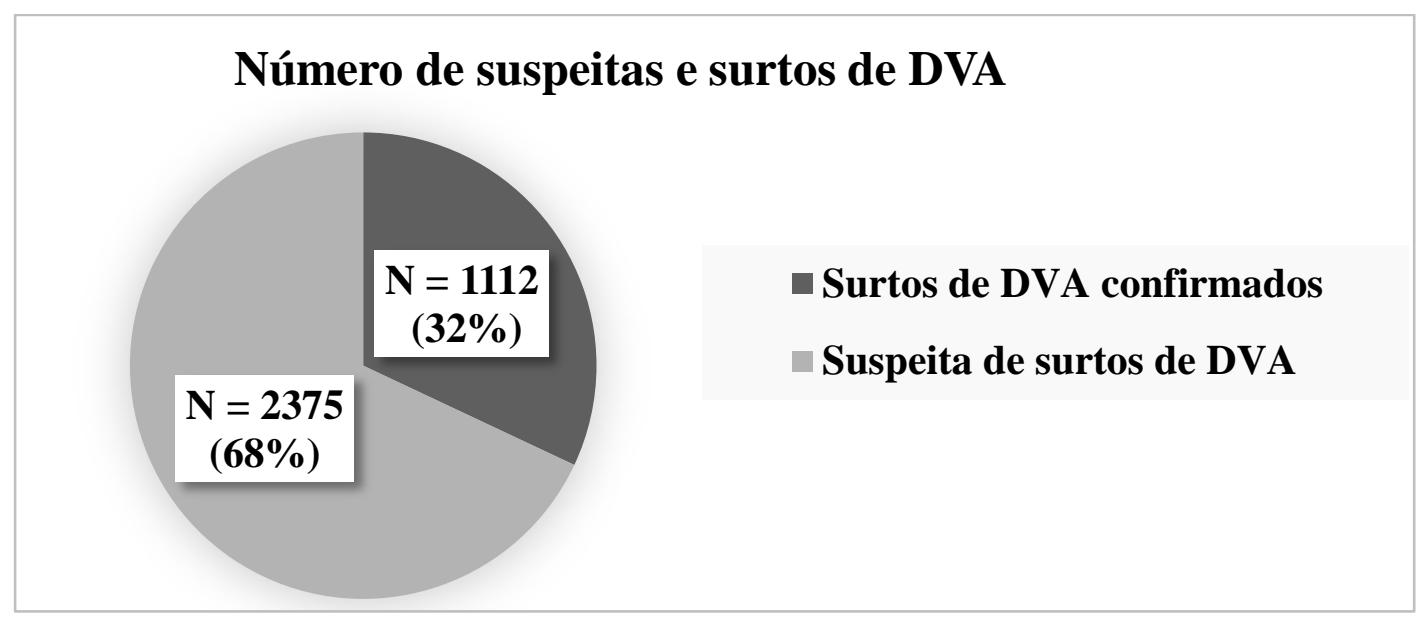

Figura 1 - Suspeitas e surtos de DVA confirmados no Estado de São Paulo, entre os anos de 2011 e 2018. 
Segundo Santos (2019), falhas na investigação podem afetar a geração de informações que direcionam a identificação dos prováveis agentes implicados, e consequentemente, a confirmação dos casos, o que compromete a caracterização epidemiológica dos surtos. O autor afirma ainda que poucos casos são notificados aos órgãos de inspeção de alimentos e às agências de saúde, e muitos dos que são notificados são escassos de informações fidedignas, e sendo assim, as informações existentes podem não apresentar a verdadeira magnitude do problema.

A Figura 2 mostra as taxas de incidência ano a ano de casos de DVA no Estado de São Paulo. Os anos de maior e menor incidência de casos foram 2011 (23,1 casos por 100 mil habitantes) e 2016 (3,46 casos por 100 mil habitantes), respectivamente.

A análise apontou tendência significativa $(\mathrm{P}=0,0126)$ de queda (coeficiente $=-0,2339)$ nas taxas de incidência de DVA no período estudado.

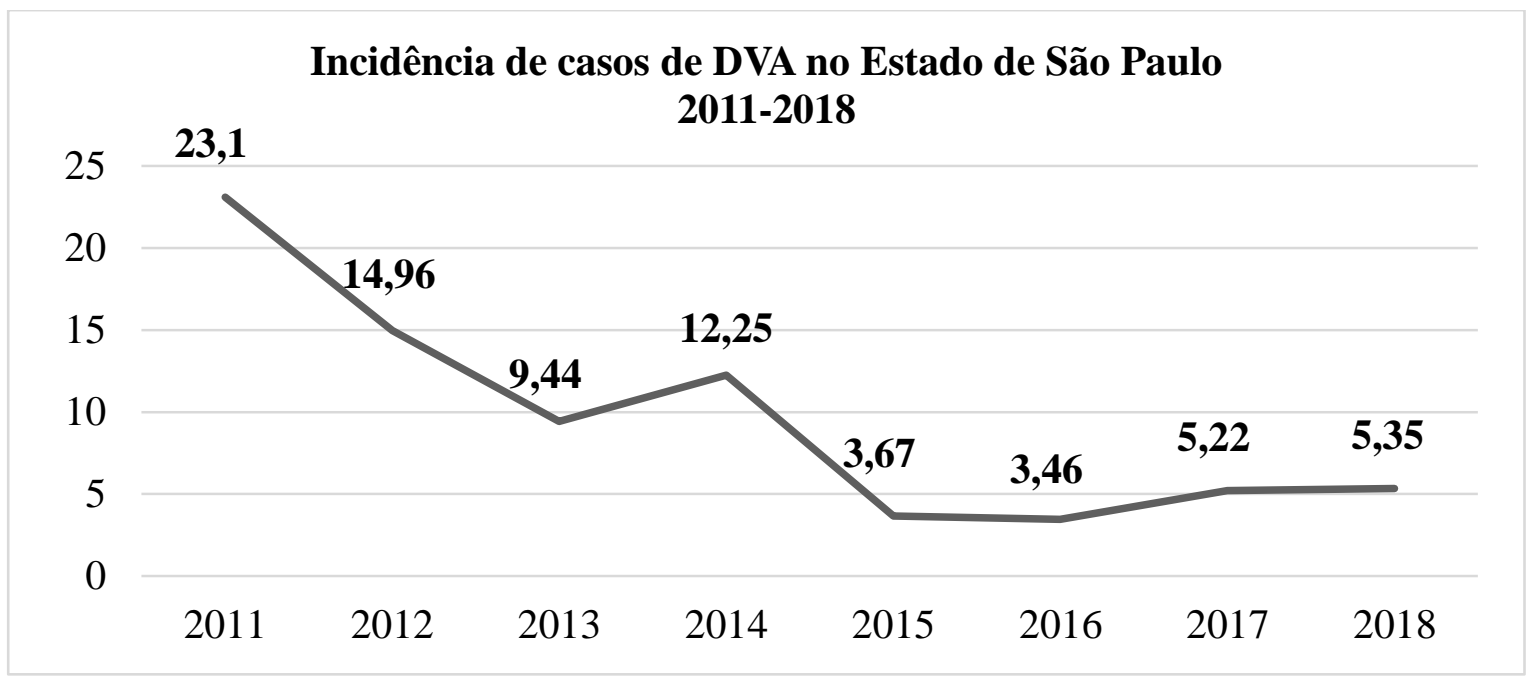

Figura 2 - Taxa de incidência de casos de DVA a cada 100 mil habitantes no Estado de São Paulo, 2011 a 2018.

Ao analisar os dados da Figura 3, pode-se afirmar que $19,45 \%$ dos surtos de DVA notificados no Brasil durante o período de 2011 a 2018 são oriundos do Estado de São Paulo. Garcia \& Duarte
(2014) afirmam que o referido Estado possui o maior número de surtos de DVA notificados, o que pode significar maior sensibilidade do sistema de vigilância epidemiológica local. Porém tal afirmação 
pode ser contestada se considerado que a população do Estado de São Paulo corresponde a $21,86 \%$ da população do país, e dessa forma se espera uma participação equivalente a esse valor na ocorrência de qualquer doença, e sendo assim, a ocorrência de DVA no Estado de São Paulo não se encontra distante do esperado considerando o tamanho da população (IBGE, 2020).

Alguns fatores de risco influenciam a predisposição do indivíduo no desenvolvimento de uma DVA. Em relação aos patógenos, tem-se a dose ingerida e a virulência; quanto ao hospedeiro, idade, estado imunológico, higiene pessoal e fatores genéticos; já a dieta tem importância em casos de deficiências nutricionais, ingestão de gorduras ou alimentos tamponados. A suscetibilidade para adquirir DVA é geral, mas crianças, idosos e imunodeprimidos têm suscetibilidade aumentada (MELO et al., 2018).

Oliveira \& Barata (2013), ao descreverem a ocorrência de surtos de DVA no Estado de São Paulo no período de 2008 a 2010, constataram que a maior taxa média de incidência de DVA no período ocorreu em crianças entre 1 e 4 anos (62,2 casos por 100.000 habitantes). Constatou-se no presente trabalho que no período de 2011 a 2018 a maior taxa média de incidência de DVA também ocorreu em crianças entre 1 e 4 anos (30,1 casos por 100 mil habitantes).

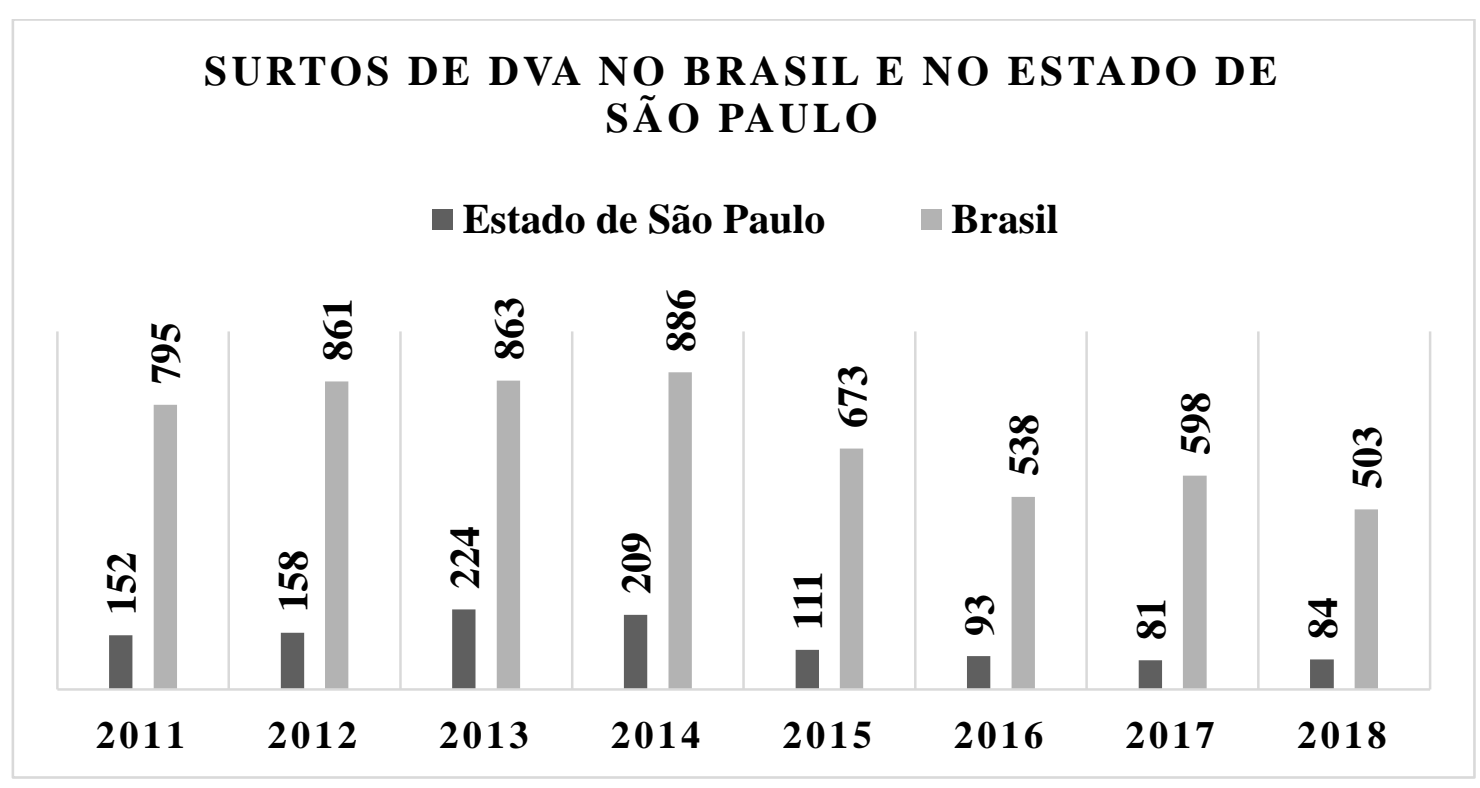

Figura 3 - Surtos de DVA ocorridos por ano, no Brasil e no Estado de São Paulo, entre os anos de 2011 e 2018. 
As demais faixas etárias da população apresentaram as seguintes taxas de médias de incidência: pacientes entre 5 e $19 \operatorname{anos}(7,38$ casos por 100 mil habitantes), pacientes entre 20 e 49 anos (6,49 casos por 100 mil habitantes) e pacientes de ou acima 50 anos (3,29 por 100 mil habitantes). Em 8.569 (25,67\%) dos casos não foi possível identificar a idade do paciente. Em relação ao sexo do paciente acometido por DVA no período, $13.290(39,81 \%)$ eram pacientes do sexo feminino e $10.325(30,94 \%)$ do sexo masculino. Em 9.762 (29,25\%) dos casos de DVA não foi registrado na base de dados o sexo do paciente.

Dentre as 17 DRS do Estado de São Paulo, a DRS IV, que notifica os surtos de DVA ocorridos na região da Baixada Santista, foi responsável pela maior incidência média de casos (50,2 casos por 100 mil habitantes) de DVA confirmados no Estado no período de 2011 a 2018, seguida pela DRS V - Barretos, que apresentou uma incidência média de casos de 48,9 casos por 100 mil habitantes e pela DRS XIV - São João da Boa Vista, com incidência de 30,6 casos por 100 mil habitantes.

Como se observa na Tabela 1 , algumas DRS apresentaram incidência média de casos muito baixa ou baixa quando comparadas às demais DRS, o que, de acordo com Oliveira \& Barata (2013), pode sugerir que algumas regionais não apresentam condições propícias para a ocorrência de surtos ou até mesmo o programa de vigilância de surtos não está plenamente implantado, não conseguindo captar todos os casos ocorridos, gerando subnotificação dos surtos de DVA.

Com o objetivo de controlar nacionalmente os fatores de ocorrência de DVA em estabelecimentos comercializadores de alimentos, foi publicada, em 15 de setembro de 2004, a RDC no. 216, que aprovou o Regulamento Técnico de Boas Práticas para Serviços de Alimentação (Brasil, 2004). O regulamento exige dos estabelecimentos produtores e manipuladores de alimentos a implantação de Boas Práticas de Fabricação, com o intuito de garantir a qualidade sanitária e a conformidade dos produtos alimentícios. 
Tabela 1 - Média de casos e incidência de surtos de DVA por DRS no Estado de São Paulo, 2011 a 2018.

\begin{tabular}{lcc}
\hline Departamento Regional de Saúde & Média de casos/ano & Incidência média /100 mil hab. \\
\hline DRS I - Grande São Paulo & 1655 & 8,4 \\
DRS II - Araçatuba & 11 & 1,52 \\
DRS III - Araraquara & 79 & 8,6 \\
DRS IV - Baixada Santista & 836 & 50,2 \\
DRS V - Barretos & 200 & 48,9 \\
DRS VI - Bauru & 96 & 5,9 \\
DRS VII - Campinas & 237 & 5,9 \\
DRS VIII - Franca & 2 & 0,3 \\
DRS IX - Marilia & 16 & 1,5 \\
DRS X - Piracicaba & 78 & 5,5 \\
DRS XI - Presidente Prudente & 19 & 2,65 \\
DRS XII - Registro & 6 & 2,2 \\
DRS XIII - Ribeirão Preto & 115 & 8,65 \\
DRS XIV - São João da Boa Vista & 237 & 30,6 \\
DRS XV - São José do Rio Preto & 241 & 16,4 \\
DRS XVI - Sorocaba & 93 & 4,15 \\
DRS XVII - Taubaté & 95 & 4,2 \\
\hline & & \\
& 63 & \\
\hline
\end{tabular}

Todavia, apesar da aplicação da legislação, muitos surtos de DVA ainda são originados nestes estabelecimentos, sendo no presente trabalho os estabelecimentos comercializadores de alimentos responsáveis por 244 (21,95\%) surtos de DVA confirmados (Figura 4).

$$
\text { Creches/escolas } \quad(27,16 \%) \quad \mathrm{e}
$$
residências $(25,63 \%)$ foram outros dois locais de maior ocorrência de surtos de
DVA, entre 2011 e 2018 no Estado de São Paulo. Observa-se na Figura 4 que ainda apareceram na lista, como locais de importância para ocorrência de DVA, refeitórios de empresas e indústrias $(7,1 \%)$, hospitais $(6,92)$, presídios $(5,21 \%)$, eventos $(3,42 \%)$ e asilos e casas de repouso $(2,34 \%)$.

Semelhante ao presente trabalho, Marinho et al. (2015), ao levantarem dados 
epidemiológicos sobre a ocorrência de DVA e seus fatores causais na Zona da Mata Sul de Pernambuco, no período de 2006 a 2013, constataram que $25 \%$ dos surtos ocorreram em creches/escolas. Silva (2009) afirma que surtos de DVA nesses locais normalmente acontecem pelo fato de que em creches e escolas a mesma refeição é produzida e consumida por um número muito maior de pessoas, sem contar que essas pessoas estão no limite da faixa de risco. Já Silva (2017) relatou que durante os anos de 1999 a 2005 foram notificados
241 surtos de DVA no Estado do Pernambuco e destes, $35,1 \%$ ocorreram em residências.

Garcia \& Duarte (2014), ao apresentarem o perfil epidemiológico dos surtos de doenças veiculadas por alimentos no Brasil no período de 1998 a 2011, também constataram que residências são locais de importância para a ocorrência de surtos de DVA, e associaram os casos à possível falta de conhecimento das Boas Práticas de Preparação dos alimentos pelos manipuladores.

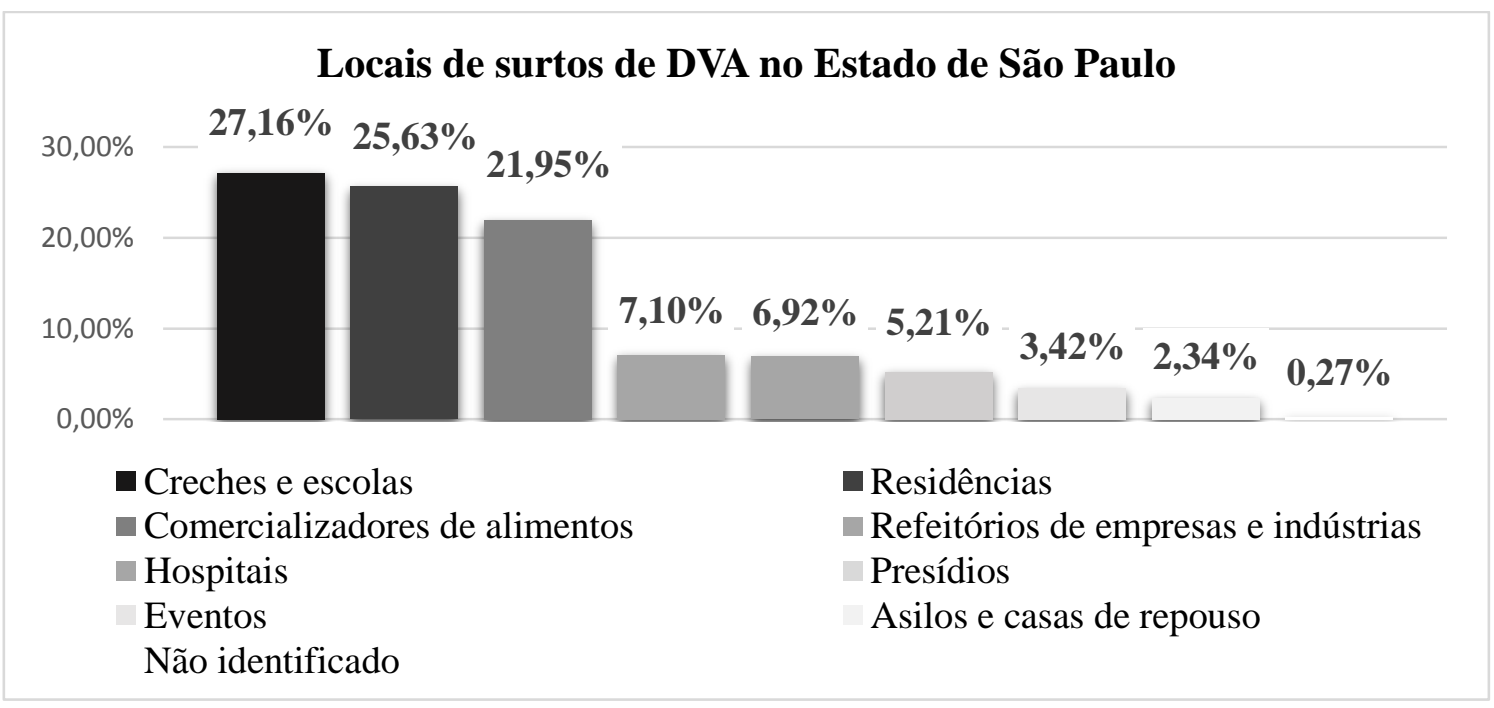

Figura 4 - Locais de surtos de DVA no Estado de São Paulo, entre os anos de 2011 e 2018.

Segundo Klein et al. (2017), os surtos ocorridos nos domicílios podem ser justificados pela predominância das refeições realizadas em casa, principalmente em municípios de pequeno e médio porte. De acordo com os autores, contribuem para a ocorrência de surtos de DVA em residências a manipulação inadequada dos alimentos, as condições higiênicas inadequadas, a falta de cuidados e conhecimento referente a temperatura e cocção, como também os microrganismos naturalmente existentes nos alimentos.

Os estabelecimentos comercializadores de alimentos são responsáveis pelo terceiro maior número 
de surtos de DVA confirmados no período de 2011 a 2018, no Estado de São Paulo. Segundo Cunha \& Amichi (2014), a ocorrência de DVA nesses estabelecimentos pode estar relacionada à urbanização crescente e às mudanças de hábitos e estilos de vida, como, por exemplo, o hábito de consumir alimentos prontos e semiprontos fora do domicílio.

De acordo com Santos (2019), a falta de capacitação dos manipuladores de alimentos nesses locais também contribui para que os estabelecimentos

podem apresentar menor número de casos de surtos simplesmente pelo fato de estarem em menor quantidade na região, o que justificaria o menor número de surtos de DVA em hospitais, uma vez que há muito mais escolas, restaurantes e domicílios no Estado do que Unidades de Saúde.

Não foi possível identificar o modo de transmissão ocorrido em 120 (10,80\%) surtos de DVA confirmados no período (Figura 5). Nascimento et al. (2016) relataram que a falta de identificação do modo de transmissão das DVA pode estar relacionada à demora na notificação do surto e à dificuldade em entrar em contato com os comensais envolvidos, bem como a falta de informações precisas sobre o surto.

Em relação aos principais alimentos apontados como veículos de doenças, comercializadores de alimentos sejam responsáveis por surtos de DVA.

Ainda observando a Figura 4, podese afirmar que em unidades hospitalares a ocorrência de surtos confirmados de DVA apresentou um número consideravelmente menor quando comparado aos demais locais.

Isso poderia ser explicado pela existência de vários critérios estabelecidos na alimentação hospitalar, do ponto de vista higiênico e sanitário, que reduzem a ocorrência de DVA. Além disso, é necessário considerar que alguns locais destacam-se as massas (13,76\%), a água $(10,70 \%)$, os múltiplos alimentos $(8,10 \%)$, o frango $(7,10 \%)$, a carne bovina $(6,65 \%)$ e os peixes e frutos do mar (4,59\%). Diferente do presente trabalho, Klein et al. (2017), ao elucidarem o perfil epidemiológico dos surtos confirmados de DVA no Rio Grande do Sul - RS, constataram que a água e as massas eram alimentos pouco envolvidos nos casos de surtos de DVA daquele Estado.

Por outro lado, os autores constaram que produtos cárneos estavam presentes em $26,13 \%$ dos surtos de DVA estudados, porcentagem semelhante à encontrado no presente trabalho, onde 204 $(18,34 \%)$ surtos de DVA foram causados por produtos cárneos (ave, carne bovina e peixes). 


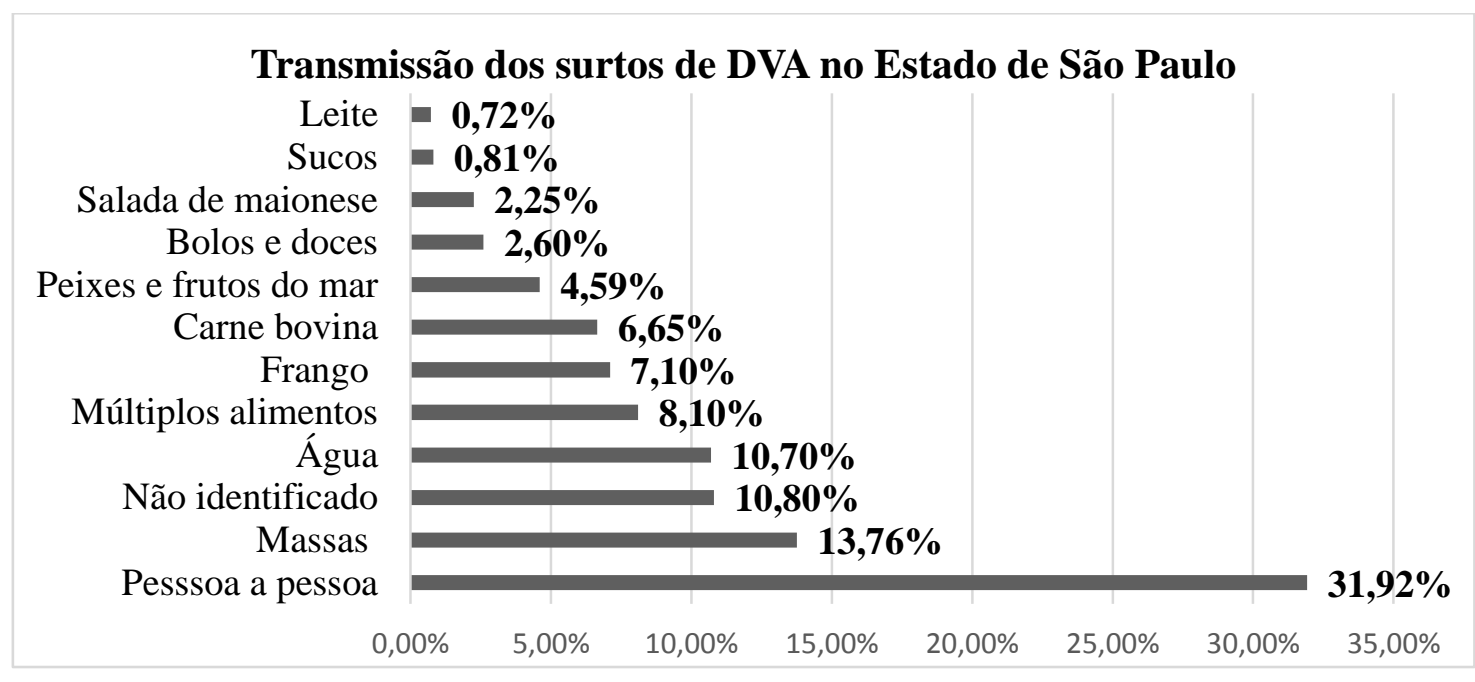

Figura 5 - Modo de transmissão dos surtos de DVA ocorridos no Estado de São Paulo, entre os anos de 2011 e 2018.

Melo et al. (2018) afirmaram que os produtos cárneos apresentam condições favoráveis à proliferação de microrganismos devido à variedade de nutrientes, umidade e à baixa acidez. Além
A transmissão de algumas DVA ainda não está bem esclarecida, porém se sabe que alguns patógenos de importância para a ocorrência de surtos, além de serem veiculados nos alimentos, podem também serem disseminados pessoa a pessoa. Esse meio de transmissão normalmente ocorre por contato de fezes ou dedos sujos de uma pessoa infectada com a boca de um indivíduo sadio. Isso acontece quando os hábitos básicos de higiene e lavagem das mãos são inadequados, e é mais comum nas famílias e ambientes infantis, especialmente entre as crianças que ainda não possuem controle esfincteriano (KAMIOKA, 2018). disso, as carnes podem ser facilmente contaminadas durante o abate do animal, evisceração, manipulação no processamento e estocagem inapropriada.

A transmissão de forma direta pessoa para pessoa foi responsável pela ocorrência de 355 (31,92\%) surtos de DVA analisados no presente estudo.

Kamioka (2018), após descrever os surtos de gastroenterite por Norovírus provenientes da vigilância epidemiológica das DVA, afirmou que a transmissão direta pessoa para pessoa é a principal forma de transmissão da infecção por esse agente, que no presente trabalho foi responsável por $146(13,14 \%)$ surtos de DVA.

Conforme a Tabela 2, considerando o número de surtos, além do Norovírus, foram identificados no presente trabalho outros agentes de 
importância na ocorrência de DVA, destacando-se entre eles o Rotavírus presente em 139 (12,51\%) surtos, a
Escherichia coli presente em 79 (8,21\%) surtos e o Vírus da Hepatite A presente em $44(3,96 \%)$ surtos de DVA.

Tabela 2 - Agentes etiológicos identificados em surtos de DVA no Estado de São Paulo, 2011-2018.

\begin{tabular}{|c|c|c|c|c|c|c|c|c|c|c|}
\hline Agente etiológico & 2011 & 2012 & 2013 & 2014 & 2015 & 2016 & 2017 & 2018 & Total & Percentual \\
\hline Não identificado & 85 & 101 & 161 & 133 & 78 & 67 & 18 & 11 & 654 & $57,06 \%$ \\
\hline Norovírus & 16 & 20 & 14 & 22 & 7 & 6 & 35 & 26 & 146 & $13,14 \%$ \\
\hline Rotavírus & 19 & 5 & 14 & 14 & 6 & 8 & 41 & 32 & 139 & $12,51 \%$ \\
\hline Escherichia coli & 15 & 3 & 4 & 7 & 9 & 2 & 12 & 27 & 79 & $8,21 \%$ \\
\hline Vírus da Hepatite A & 15 & 7 & 7 & 10 & 0 & 1 & 1 & 3 & 44 & $3,96 \%$ \\
\hline Salmonella spp. & 4 & 8 & 6 & 5 & 1 & 1 & 3 & 3 & 31 & $2,79 \%$ \\
\hline Coliformes & 1 & 4 & 4 & 8 & 4 & 2 & 3 & 3 & 29 & $2,61 \%$ \\
\hline Bacillus cereus & 3 & 3 & 6 & 5 & 4 & 0 & 1 & 3 & 25 & $2,25 \%$ \\
\hline Staphylococcus spp. & 6 & 3 & 3 & 1 & 4 & 2 & 0 & 1 & 20 & $1,8 \%$ \\
\hline Clostridium botulinum & 0 & 3 & 3 & 3 & 3 & 3 & 3 & 1 & 19 & $1,71 \%$ \\
\hline Shigella spp. & 0 & 0 & 4 & 5 & 2 & 0 & 2 & 0 & 13 & $1,17 \%$ \\
\hline $\begin{array}{l}\text { Provável } \\
\text { escombroide }\end{array}$ & 1 & 0 & 1 & 1 & 0 & 1 & 0 & 0 & 4 & $0,36 \%$ \\
\hline Cryptococcus spp. & 1 & 0 & 0 & 1 & 0 & 0 & 1 & 0 & 3 & $0,27 \%$ \\
\hline Giárdia & 0 & 0 & 0 & 0 & 0 & 0 & 2 & 0 & 2 & $0,18 \%$ \\
\hline Entamoeba histolytica & 0 & 0 & 0 & 0 & 1 & 0 & 0 & 1 & 2 & $0,18 \%$ \\
\hline Toxoplasma gondii & 0 & 0 & 0 & 0 & 0 & 0 & 0 & 2 & 2 & $0,18 \%$ \\
\hline Toxina botulínica & 0 & 1 & 0 & 0 & 0 & 0 & 0 & 0 & 1 & $0,09 \%$ \\
\hline Campylobacter jejuni & 0 & 0 & 0 & 0 & 0 & 1 & 0 & 0 & 1 & $0,09 \%$ \\
\hline Dinophysis spp. & 0 & 0 & 0 & 0 & 0 & 1 & 0 & 0 & 1 & $0,09 \%$ \\
\hline
\end{tabular}

Ferreira (2017), ao analisar a evolução das Doenças Veiculadas por Alimentos nas diferentes regiões do Brasil entre 2010 e 2015, constatou que em $57,8 \%$ dos surtos não foi possível realizar a identificação do agente etiológico 
responsável pela DVA, resultado semelhante ao encontrado no presente trabalho, no qual não foi possível realizar a identificação do agente etiológico em 654 $(57,06 \%)$ dos 1112 surtos de DVA confirmados.

Foram notificados, entre 2011 e 2018 no Estado de São Paulo, 11 óbitos por DVA, sendo a Salmonela spp. (36,36\%), a Escherichia coli $(18,19 \%)$, o Rotavírus (9,09\%) e a Shigella spp. $(9,09 \%)$ os agentes etiológicos responsáveis pelos surtos que ocasionaram os óbitos.
Em 27,27\% dos óbitos não foi possível identificar o agente etiológico responsável pela DVA (Figura 6).

O quadro clínico do paciente acometido por uma DVA depende do agente etiológico envolvido, variando desde leve desconforto intestinal até quadros mais severos, com desidratação grave, diarreia sanguinolenta, insuficiência renal aguda (síndrome hemolítica urêmica) e insuficiência respiratória (botulismo). Alergia por hipersensibilidade individual a certos alimentos não é considerada uma DVA (Brasil, 2019).

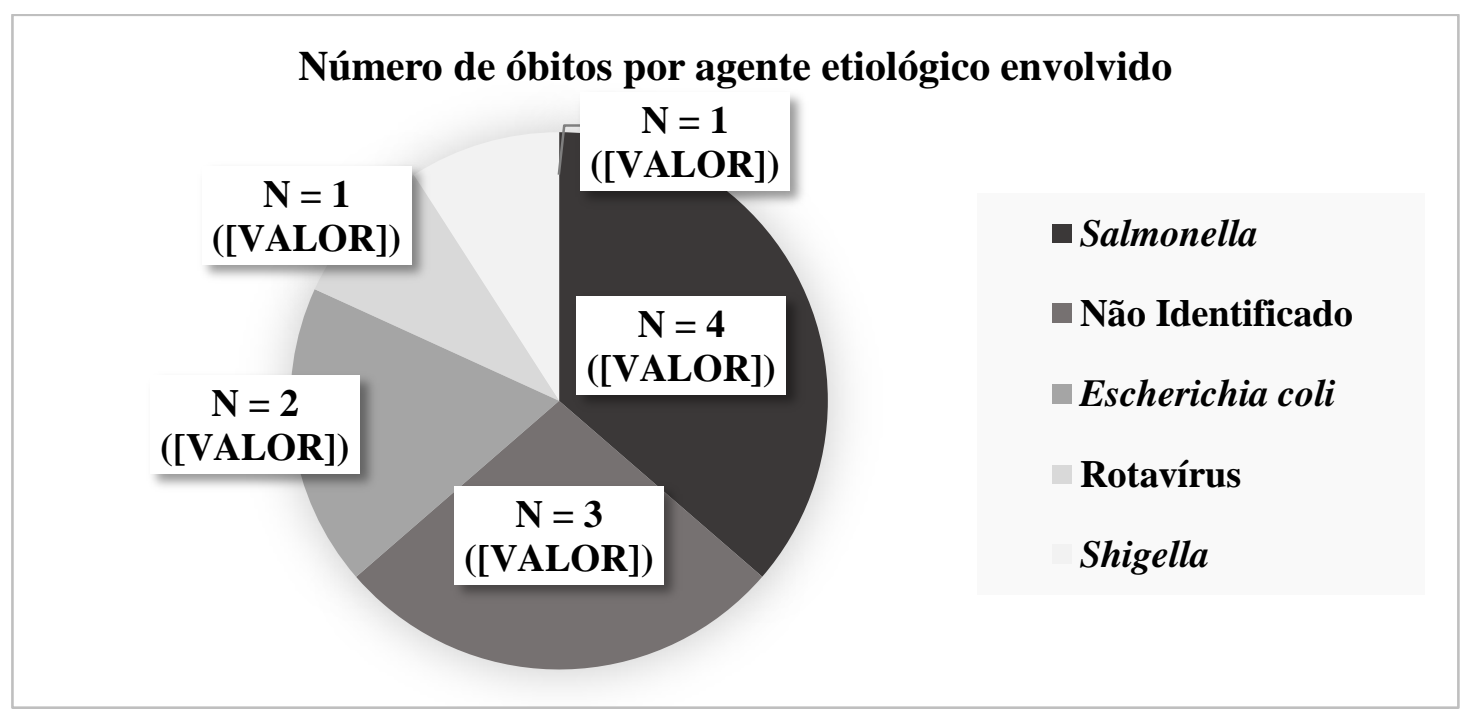

Figura 6 - Número de óbitos por agente etiológico envolvido nos surtos de DVA ocorridos no Estado de São Paulo, entre os anos de 2011 e 2018.

Em $1057(95,06 \%)$ surtos os pacientes apresentaram quadros de diarreia, em $47(4,22 \%)$ surtos os pacientes foram acometidos por Hepatite
A, em $5(0,54 \%)$ surtos os pacientes apresentaram quadros de intoxicação, em $1(0,09 \%)$ surto os pacientes apresentaram botulismo e em $1(0,09 \%)$ outro os 
pacientes apresentaram febre tifoide.

Um surto de DVA pode ser encerrado por critério laboratorial clínico, laboratorial bromatológico, laboratorial clínico e bromatológico ou por critério clínico epidemiológico (Brasil, 2010).

No presente trabalho, $515(46,32 \%)$ surtos de DVA foram encerrados por critério clínico epidemiológico, 298 $(26,80 \%)$ surtos foram finalizados por análise laboratorial das amostras clínicas, $50(4,49 \%)$ surtos se encerraram por meio da análise laboratorial das amostras bromatológicas e $20(1,80 \%)$ surtos foram encerrados pela realização de ambas as análises, das amostras clínicas e das amostras bromatológicas. Contudo, em 229 (20,59\%) dos surtos, a informação do critério de encerramento do surto encontrase inconclusiva na base de dados.

\section{Conclusões}

Conclui-se no presente trabalho que as regiões da Baixada Santista, de Barretos e de São João da Boa Vista apresentaram as maiores incidências médias de casos de DVA no de Estado de São Paulo, no período de 2011 a 2018. Os locais de maior ocorrência de surtos foram creches e escolas, residências e estabelecimentos comercializadores de alimentos.

Os alimentos mais envolvidos nos surtos de DVA foram massas, água e produtos cárneos, porém a transmissão direta pessoa para pessoa foi responsável pela maioria dos casos. Os agentes etiológicos de destaque na ocorrência de surtos no Estado foram o Norovírus e o Rotavírus, considerando o número de casos e surtos, e a Salmonella spp., a Escherichia coli e a Shigella spp., considerando o número de óbitos. A população feminina foi a mais acometida, e crianças de 1 a 4 , a faixa etária com maior incidência de casos.

Por fim, vale ressaltar que informações importantes sobre os casos de DVA, como os alimentos e agentes etiológicos mais envolvidos nos surtos, sexo dos pacientes acometidos e critério de encerramento dos casos, encontram-se incompletas na base de dados, o indica falhas nos processos de notificação e investigação dos surtos ou na alimentação do sistema, prejudicando dessa forma o conhecimento da evolução dos surtos de DVA no Estado de São Paulo.

\section{Referências}

AMARAL, F.L.E. do., SILVA, E.F. da., LACERDA, F.A.L., PINTO, N.B. Análise retrospectiva dos casos de intoxicação humana por alimentos no Brasil no período de 2008 a 2016. RevInter, v. 12, n. 1, 2019.

BRASIL. Ministério da Saúde. Agência Nacional de Vigilância Sanitária. Resolução RDC $\mathrm{n}^{\circ}$ 216, de 15 de setembro de 2004. Dispõe sobre regulamento técnico de boas práticas para serviços de alimentação. Diário Oficial da União. Brasília, 2004. 
BRASIL. Ministério da Saúde. Surtos de doenças transmitidas por alimentos no Brasil no ano de 2018. Informe Anual de 2018. Disponível em: https://portalarquivos2.saude.gov.br/images/pd f/2019/fereveiro/15/Apresenta----o-SurtosDTA---Fevereiro-2019.pdf. Acessado em: 28 de março de 2020.

CIRNE, L.S.F. de., CABRERA, P. G. J. Ações em Saúde Única para redução de parasitoses infantis: revisão integrativa de literatura. Revista Saber Digital, v. 12, n. 2, p. 136-149, jan. 2020.

CUNHA, L.F.; AMICHI, K.R. Relação entre a ocorrência de enteroparasitoses e práticas de higiene de manipuladores de alimentos: revisão da literatura. Revista Saúde e Pesquisa, v. 7, n. 1, p. 147- 157, jan./abr. 2014.

GARCIA, D.P., DUARTE, D.A. Perfil epidemiológico de surtos de doenças transmitidas por alimentos ocorridos no Brasil. Revista Eletrônica Acervo Saúde/Electronic Journal Collection Health, v. 2178, p. 2091, 2014.

DOHOO, I.; MARTIN, W.; STRYHN, H. Veterinary epidemiologic research. 2 ed. AVC Inc., 2010. 865 p.

FERREIRA, A.F.J. de. Panorama das doenças transmitidas por alimentos no Brasil entre 2000 e 2015. 2017. Tese de Doutorado (Nutrição em Saúde Pública). Universidade de São Paulo. São Paulo, 2017.

IBGE. Instituto Brasileiro de Geografia e Estatística. Pesquisa Nacional por Amostra de Estados. Estado de São Paulo, 2020.

KAMIOKA, A. G. Norovírus: principal causa de gastroenterite epidêmica no município de São Paulo. Tese de Mestrado (Epidemiologia). Universidade de São Paulo. São Paulo, 2018.
KLEIN, L.R., BISOGNIN, R.P., FIGUEIREDO, M.S.D. Estudo do Perfil Epidemiológico dos Surtos de Doenças de Transmissão Hídrica e Alimentar no Rio Grande do Sul: uma revisão dos registros no Estado. Hygeia-Revista Brasileira de Geografia Médica e da Saúde, v. 13, n. 25, p. 48-64, 2017.

MARINHO, G.A., OLIVEIRA, G.S. de, LIMA, J.L. de, LOPES, W.M.A., NUNES, G.A., NUNES, M.G.A. Perfil epidemiológico das doenças transmitidas por alimentos e seus fatores causais na região da zona da mata sul de Pernambuco. Journal of Health Sciences, v. 17, n. 4, 2015.

MELO, E.S. de., AMORIN, W.R., PINHEIRO, R.E.E., CORREIA, P.G.N., CARVALHO, S.M.R., SANTOS, A.R.S., SOUZA, F.V. Doenças transmitidas por alimentos e principais agentes bacterianos envolvidos em surtos no brasil: revisão. Publicações Veterinária, v. 12, p. 131, 2018.

NASCIMENTO, I. R. do., SENA, T. L. de., CASTRO, F. F. (2016). Doenças Veiculadas por Alimentos: estudo da qualidade microbiológica de amostras de leite, ovos, carnes bovina e de frango encontradas em algumas feiras e açougues no Distrito Federal. Programa de Iniciação CientíficaPIC/UniCEUB-Relatórios de Pesquisa, v. 1, n. $1,2016$.

OLIVEIRA, M.E.B.D., BARATA, R.D.C.B. (2013). Surtos de Doenças Transmitidas por Alimentos no Estado de São Paulo, 20082010. BEPA. Boletim Epidemiológico Paulista (Online), 10 (109), 18-18.

SANTOS, J.M.D. Casos de intoxicações por alimentos e bebidas notificados em Barra do Garças, Mato Grosso. Monografia (Bacharelado em Farmácia). Universidade Federal de Mato Grosso. Instituto de Ciências Biológicas da Saúde. Centro Universitário do Araguaia. Barra do Garças, 2019. 
Antônio et al., Revista Brasileira de Higiene e Sanidade Animal (v.15, n.2) p. 1 - 16 abr - jun (2021)

SILVA, G.C.J. de. Incidência de doenças transmitidas por alimentos (DTA) no estado de Pernambuco, um acompanhamento dos dados epidemiológicos nos últimos anos. Caderno de Graduação-Ciências Biológicas e da SaúdeUNIT-PERNAMBUCO, v. 3, n. 1, p. 23, 2017.

SILVA, Y. da. Doenças transmitidas por alimentos no município do Rio de Janeiro: perfil epidemiológico e controle. Tese de Mestrado (Vigilância Sanitária). Instituto Nacional de Controle de Qualidade em Saúde da Fundação Oswaldo Cruz (FIOCRUZ). Rio de Janeiro, 2009.

$\begin{array}{lrl}\text { WORLD } & \text { HEALTH } & \text { ORGANIZATION } \\ \text { (WHO). } & \text { Foodborne } & \text { disease burden }\end{array}$
epidemiology reference group 2007-2015, who estimates of the global burden of foodborne diseases, 2015. Disponível em: https://apps.who.int/iris/bitstream/handle/1066 5/199350/9789241565165_eng.pdf?sequence $=$ 1. Acessado em: 28 de março de 2020. 